\title{
Hybrid Simulation Study on Anisotropic Response of Ions to a Low-Frequency Wave in a Field-Reversed Configuration
}

\author{
Takahiro URANO, Toshiki TAKAHASHI, Akiyoshi HOSOZAWA ${ }^{1)}$, \\ Tomohiko ASAI ${ }^{1)}$ and Shigefumi OKADA ${ }^{2)}$ \\ Graduate School of Science and Technology, Gunma University, Kiryu 376-8515, Japan \\ ${ }^{1)}$ College of Science and Technology, Nihon University, Tokyo 101-8308, Japan \\ ${ }^{2)}$ Osaka University, Suita 565-0871, Japan
}

(Received 24 January 2019 / Accepted 10 February 2019)

\begin{abstract}
Using a hybrid simulation code, we studied the application of low-frequency wave to a field-reversed configuration plasma. The wave application antenna was arranged to sandwich the separatrix, and the antenna current was varied sinusoidally with a maximum of $30 \mathrm{kA}$ and a frequency of $80 \mathrm{kHz}$. The simulation revealed that when the ion pressure is regarded as a tensor, the parallel ion temperature estimated from the axial velocity distribution near the midplane exceeds the perpendicular temperature. That is, the plasma ions respond anisotropically to a low-frequency wave.
\end{abstract}

(c) 2019 The Japan Society of Plasma Science and Nuclear Fusion Research

Keywords: field-reversed configuration, low-frequency wave, ion heating, hybrid simulation, anisotropic ion temperature

DOI: $10.1585 /$ pfr. 14.1203064

A field-reversed configuration (FRC) plasma [1] exists in an extremely high-beta state and has a field-null point inside the separatrix. This suggests that some real phenomena cannot be clarified by magnetohydrodynamic simulations [2-5]. For this reason, many recent studies have applied the ion-particle model [6-8] to reproduce the particle effects of ions with Larmor radii as large as the major radius of the FRC plasma. In recent years, TAE Technologies in the USA has extended the FRC lifetime by various control techniques [9]. Effective nuclear fusion reactions also require an ion heating technique. However, conventional heating techniques such as ion cyclotron range of frequencies heating is supposed to be inapplicable in this case, because of the narrow resonance region in a high-beta FRC plasma with strong non-uniformity. Instead, low-frequency waves have been anticipated for ion heating of FRC, and experiments have been conducted on the FIX machine $[10,11]$. Ion heating effects were demonstrated in these experiments. In addition, magnetic pumping by a low-frequency wave was recently proposed to heat plasma ions [12]. Therefore, to elucidate the ion heating mechanisms and the feasibility of ion heating, the plasma response to a low-frequency wave should be investigated by a non-linear simulation method. In this brief report, we present the results of initial 3-dimensional (3-d) hybrid simulations (regarding the ions in plasma as particles and the electrons as fluid) of an FRC plasma subjected to lowfrequency wave heating. The anisotropic responses of the ions are revealed in their velocity distribution.

The simulations were performed in a 3-d Cartesian

author'se-mail: t09306013@gunma-u.ac.jp coordinate system. The calculation region was sized $(x, y, z)=( \pm 0.4 \mathrm{~m}, \pm 0.4 \mathrm{~m}, \pm 1.0 \mathrm{~m})$. The initial equilibrium was obtained from the Grad-Shafranov equation, and the external magnetic field was set to $0.05 \mathrm{~T}$. The initial ion temperature and the electron temperature were set to $60 \mathrm{eV}$ and $30 \mathrm{eV}$, respectively. Both parameters were constant in the separatrix, and decreased exponentially outside the separatrix. In the equilibrium calculation, the radius and length of the separatrix were determined as $r_{\mathrm{s}}=0.2 \mathrm{~m}$ and $l_{\mathrm{s}}=1.36 \mathrm{~m}$, respectively, and the maximum density was $6.96 \times 10^{19} \mathrm{~m}^{-3}$. The ions to be tracked were evenly distributed as super-particles within the calculation region, and were weighted such that their number followed the equilibrium density. By solving the equations of motion, we can determine the trajectory of the ions. Coulomb collisions were handled by the models in Refs. [13] and [14]. The results of the trajectory calculation were converted to fluid information by the particle-in-cell method. The spatial distribution of the ion temperature can be obtained by dividing the ion pressure tensor

$$
P_{i j}=\int m\left(v_{i}-u_{i}\right)\left(v_{j}-u_{j}\right) f \mathrm{~d} \mathbf{v},
$$

by the ion density. Here, $P_{i j}$ is the ion pressure tensor, $m$ is the ion mass, $v_{i}$ is the subscript notation for the ion velocity, $f$ is the distribution function, and $u_{i}$ is the subscript notation for the ion flow velocity. The subscripts $i$ and $j$ represent each component of $x, y$, and $z$. A low-frequency wave was generated from the antenna as described later. The equations employed in the hybrid simulation code are shown in Ref. [15]. The wave application antenna was a 




Fig. 1 Ion temperature tensors in the $r-z$ plane. Top: Initial $T_{\mathrm{i} \perp}$ and $T_{\mathrm{i} \|}$, bottom: $T_{\mathrm{i} \perp}$ and $T_{\mathrm{i} \|}$ at $27.7 \mu$ s $(10 \mu \mathrm{s}$ after the wave application). The black lines indicate the iso-flux surfaces.

loop antenna with a radius of $0.30 \mathrm{~m}$, and two loop antennas were arranged at $z= \pm 0.59 \mathrm{~m}$. The applied antenna current was sinusoidal with a maximum of $30 \mathrm{kA}$ and a frequency of $80 \mathrm{kHz}$. Its resonance point with the ion cyclotron frequency was inside the separatrix. This $30 \mathrm{kA}$ antenna current created a magnetic field of approximately $0.001-0.04 \mathrm{~T}$ in the separatrix.

In this simulation, the low-frequency wave was applied at $17.7 \mu$ s after the beginning. During this time, the ions located at the midplane $(z=0 \mathrm{~m})$ move to the axial end of the device and can be lost if their speed matches the thermal speed in the $z$ direction. Figure 1 shows the 2-d distributions of the ion temperature and isomagnetic flux lines. During the simulation, the FRC remained largely axisymmetric, so we defined the separatrix by a poloidal flux function and displayed the half cross-section of its interior. Figure 1 separately shows the ion temperatures in the directions parallel and perpendicular to the magnetic field. As the FRC is elongated and the main component of the magnetic field is the $z$ component, the ion temperatures in the perpendicular and parallel directions were simply computed as $T_{\mathrm{i} \perp} \approx\left(P_{x x}+P_{y y}\right) / 2 n_{\mathrm{i}}$ and $T_{\mathrm{i} \|} \approx P_{z z} / n_{\mathrm{i}}$ respectively, where we have used the pressure obtained by Eq. (1), and $n_{i}$ is the ion density. It can be said that these expressions hold because the magnetic field has only a $z$ component near the midplane. Observing the vicinity of the midplane in Fig. 1, we find that $T_{\mathrm{i} \perp} \approx T_{\mathrm{i} \|}$ at $0.0 \mu \mathrm{s}$, but $T_{\mathrm{i} \perp} \neq T_{\mathrm{i} \|}$ at $27.7 \mu \mathrm{s}(10 \mu \mathrm{s}$ after the wave application). For this reason, the ion velocity distribution appears anisotropic in Fig. 1. In particular, $T_{\mathrm{i} \|}$ rises in the region near the midplane and the device axis. This result clarifies that the low-frequency wave application generates anisotropy in the ion motion.

To clarify the anisotropy of the ion motion, we cal-

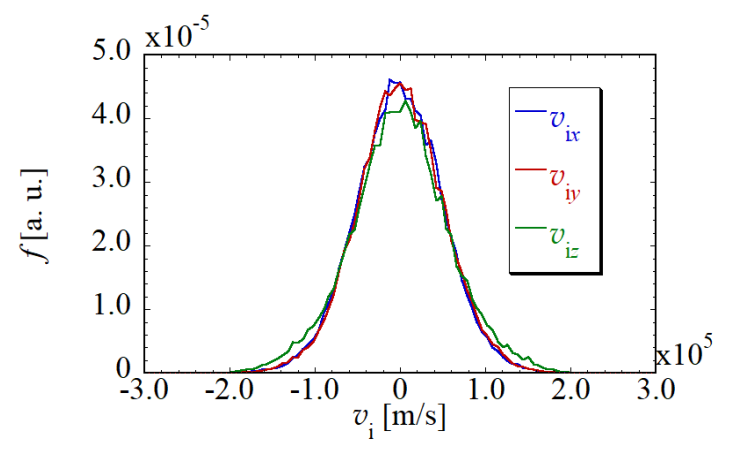

Fig. 2 Velocity distributions of the particles in the region $-0.2 \leq$ $z \leq 0.2,0 \leq r \leq 0.1$ at $27.7 \mu$ s.

culated the velocity distribution of the ions (see Fig. 2). Because a sufficient number of super-particles cannot be secured in one calculation cell to draw the velocity distribution, we prepared a dedicated ion group separate from the super-particles in the hybrid simulation. The detailed velocity distribution of these ions was obtained by solving their particle trajectories in the electromagnetic field obtained by the hybrid simulation. Figure 2 shows the velocity distribution of the particles in the range $-0.2 \leq z \leq 0.2$, $0 \leq r \leq 0.1$ at $27.7 \mu$ s. Note that the distribution widths of the $x$ and $y$ velocity components generally correspond to the $T_{\mathrm{i} \perp}$ distribution, and that of the $z$ component corresponds to the $T_{\mathrm{i} \|}$ distribution. Although the velocity distributions of the $x$ and $y$ components are not significantly different, the $z$ component shows an increase in the number of high speed particles. This result indicates an anisotropic response of the ions to the wave application.

This work is performed on "Plasma Simulator" (FUJITSU FX100) of NIFS with the support and under the auspices of the NIFS Collaboration Research program (NIFS18KNST127). This work was supported by JSPS KAKENHI Grant Number JP16K06939.

[1] M. Tuszewski, Nucl. Fusion 28, 2033 (1988).

[2] N. Iwasawa et al., Phys. Plasmas 7, 931 (2000).

[3] A. Ishida et al., Phys. Fluids B 4, 1280 (1992).

[4] R. Horiuchi and T. Sato, Phys. Fluids B 1, 581 (1989).

[5] E. Kansa et al., J. Comp. Phys. 234, 172 (2013).

[6] E.V. Belova et al., Phys. Plasmas 7, 4996 (2000).

[7] H. Ohtani et al., Phys. Plasmas 10, 145 (2003).

[8] Y.A. Omelchenko, Phys. Rev. E 92, 023105 (2015).

[9] M.W. Binderbauer et al., Phys. Plasmas 22, 056110 (2015).

[10] K. Yamanaka et al., Phys. Plasmas 7, 2755 (2000).

[11] S. Okada et al., Nucl. Fusion 41, 625 (2001).

[12] J. Egedal, Phys. Plasmas 25, 072510 (2018).

[13] M.E. Jones et al., J. Comp. Phys. 123, 169 (1996).

[14] T. Taguchi et al., Opt. Expr. 18, 2389 (2010).

[15] T. Urano et al., Plasma Fusion Res. 14, 2403022 (2019). 